\title{
A MICRO CO2 GAS SENSOR BASED ON SENSING OF PH-SENSITIVE HYDROGEL SWELLING BY MEANS OF A PRESSURE SENSOR
}

\author{
S. Herber, J. Bomer, W. Olthuis, P. Bergveld, A. van den Berg
}

$\mathrm{MESA}^{+}$Research Institute, Chair of BIOS, University of Twente

\begin{abstract}
In this paper a sensor is presented for the detection of carbon dioxide gas inside the stomach in order to diagnose gastrointestinal ischemia. The operational principle of the sensor is measuring the $\mathrm{CO}_{2}$ induced pressure generation of a confined $\mathrm{pH}-$ sensitive hydrogel by means of a micro pressure sensor. The sensor is capable of measuring $\mathrm{CO}_{2}$ with a response time between 2 and 4 minutes and a maximum pressure of $0.29 \times 10^{5} \mathrm{~Pa}$ at $20 \mathrm{kPa} \mathrm{CO}_{2}$. The sensor is able to resist up to $1 \mathrm{M} \mathrm{HCl}$ acid as can be present inside the stomach. The results are very promising for real application and clinical trials are planned.
\end{abstract}

Keywords: pH-sensitive, hydrogel, pressure, sensor, gastrointestinal ischemia

\section{INTRODUCTION}

Gastrointestinal ischemia occurs when blood flow is insufficient to deliver oxygen to the stomach and intestines [1,2]. Causes might be occlusion of arteries/veins, sepsis, or circulatory shock. Symptoms are abdominal pain, nausea, vomiting, hypotension and/or weight loss. Gastrointestinal ischemia is life-threatening.

Gastrointestinal ischemia is accompanied by an anaetobic metabolism resulting in an unusually high concentration of carbon dioxide inside the stomach and intestines. By measuring the partial pressure of carbon dioxide $\left(\mathrm{PCO}_{2}\right)$ inside the stomach it can be diagnosed whether a person has gastrointestinal ischemia. The desired measurement range of a suitable sensor is 3 to $20 \mathrm{kPa} \mathrm{CO}_{2}$ and a detection limit of $0.1 \mathrm{kPa} \quad \mathrm{CO}_{2}$ is required. Furthermore, the response time of the sensor should be far below 10 minutes and the sensor should be able to resist all stomach content and should fit in a catheter with a maximum diameter of $4.5 \mathrm{~mm}$.

Various $\mathrm{CO}_{2}$ measurement principles have been proposed in literature, however, these do not meet all our requirements. Therefore a sensor with a novel measurement method was successfully developed. The sensor exploits a hydrogel, which is a three dimensional polymer network that can contain a large amount of water. By incorporating functional groups on the polymer backbone, a hydrogel becomes stimulus-sensitive, which means that it can swell and shrink in response to changes in stimuli. Stimuli can be pH [3], temperature [3], ion concentration [4], electrical field [5], solvent composition [3], and light [6]. Several types of hydrogel have been explored for micro sensors and actuators [7]

The hydrogel we use is $\mathrm{pH}$-sensitive due to present amine groups. By decreasing the $\mathrm{pH}$, the amine groups become protonated and thus have a positive charge. As a result, negative counterions from the solution are attracted. Consequently, the ion concentration inside the hydrogel is much higher than the surrounding solution and there is an osmotic pressure difference. The hydrogel will swell until the elastic forces inside the gel are in equilibrium with the osmotic force.

In our device the hydrogel is confined between a micro pressure sensor and a porous cover, as shown in figure 1. In this situation, the hydrogel is not able to swell but will generate a pressure, which is measured by the pressure sensor. Furthermore, the porous cover contains a reservoir with bicarbonate electrolyte, which is retained by a gas permeable membrane. Water is known to react. with $\mathrm{CO}_{2}$ resulting in protons and thus a $\mathrm{pH}$ decrease,

$$
\mathrm{CO}_{2}+\mathrm{H}_{2} \mathrm{O} \Leftrightarrow \mathrm{H}^{+}+\mathrm{HCO}_{3}^{+}
$$

When bicarbonate is added to water, the change in $\mathrm{pH}$ due to $\mathrm{CO}_{2}$ is doubled [8]. This principle is exploited in our sensor.

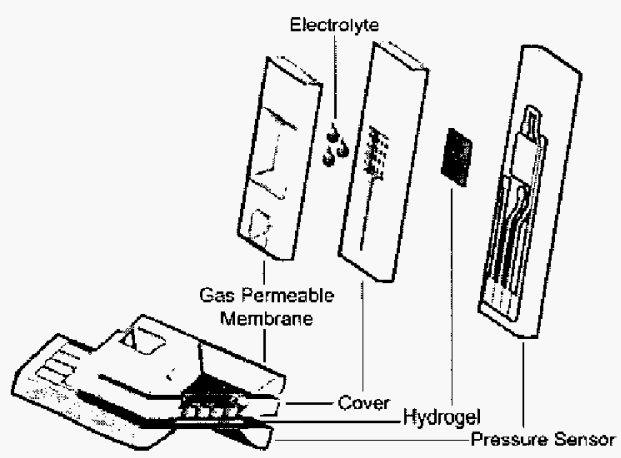

Fig.1. Schematic representation of the hydrogelbased carbon dioxide sensor.

In figure 2 the complete operational principle of the sensor is given. $\mathrm{CO}_{2}$ gas diffuses though the gas permeable membrane into the bicarbonate electrolyte. A reaction takes place resulting in a $\mathrm{pH}$ decrease. The $\mathrm{pH}$-sensitive hydrogel responds to the $\mathrm{pH}$ decrease by generating additional pressure, which is measured by the pressure sensor. Thus, the 
measured pressure is in relation with the partial pressure of $\mathrm{CO}_{2}$. Note that the process is fully reversible. Preliminary research already demonstrated the feasibility of the sensor principle $[9,10]$.

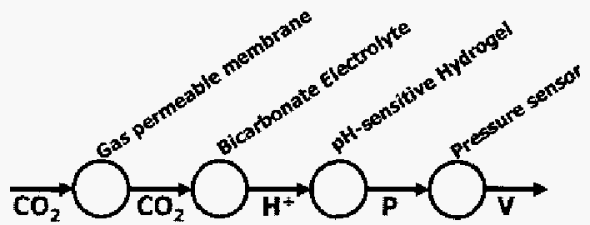

Fig. 2. Operational principle of the hydrogel-based carbon dioxide sensor.

The advantage of the sensor is that no reference electrode is required. Furthermore the sensor uses an existing pressure sensor, which facilitates the acceptance of the $\mathrm{CO}_{2}$ sensor in the medical field.

\section{EXPERIMENTAL}

Prehydrogel solution is prepared with 2bydroxyethyl methacrylate (HEMA) and dimethylaminoethyl methacrylate (DMAEMA) with a molar ratio of $95 / 5$, and solvent containing ethylene glycol and water in equimolar amounts. To the total mole amount of monomers (HEMA+DMAEMA) $\quad 1.5$ mol $\%$ cross-linker tertraethylene glycol dimethacrylate (TEGDMA) and $3 \mathrm{~mol} \%$ photoinitiator 2,2-dimethoxy-2phenylacetophenone (DMPAP) are added. The mole ratio between the monomers and the solvent is 1 to 1.2 . The solution is stored in dark at $4{ }^{\circ} \mathrm{C}$.

The electrolyte is prepared from $17 \mathrm{mM}$ sodium bicarbonate, $8 \mathrm{mM}$ sodium chloride and deionized water (DI). These concentrations are optimal with respect to the hydrogel properties as cetermined earlier [11].

The pressure sensor is acquired from Sentron Europe BV (type P4.2) and the porous cover and gas permeable -membrane, consisting of a silicon carrier and polydimethylsiloxane (PDMS), are made with standard cleanroom techniques.

The porous cover, see figure 3 , is (capillary) glued (Hysol, EE0079/HDo070, Loctite) to the pressure sensor. The porous cover contains not only a reservoir for the electrolyte but also a hydrogel cavity. After gluing, this cavity is located exactly above the pressure sensor membrane. By using vacuum, this cavity is filled with the prehydrogel solution while the pores and electrolyte reservoir are blocked by a PDMS plug. By exposure to UV light (ELC-403 light curing system, The ElectroLite Corporation) for 90 seconds the prehydrogel solution is polymerized to the actual hydrogel with a thickness of $5 \mu \mathrm{m}$. Afterwards the PDMS plug is removed. Next, the gas permeable membrane is (capillary) glued on top. Through a special filling channel the electrolyte reservoir is filled with the bicarbonate solution by means of vacuum. The special filling channel is closed by silicone glue.

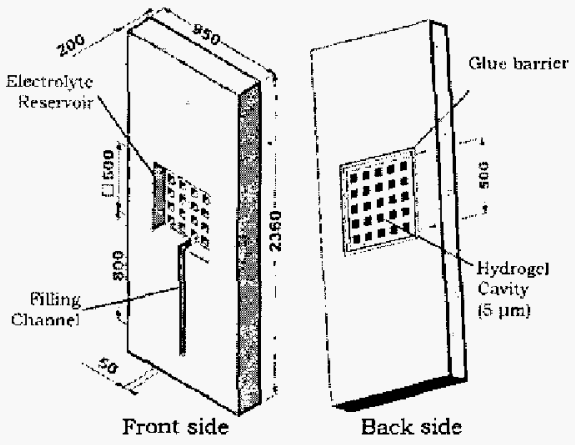

Fig. 3. Schematic of the porous cover (in $\mu \mathrm{m}$ ).

A picture of the hydrogel-based sensor is shown in figure 4. The outer dimensions are $2.92 \times 0.95 \times 0.70 \mathrm{~mm}^{3}$, which is small enough to fit in a catheter.

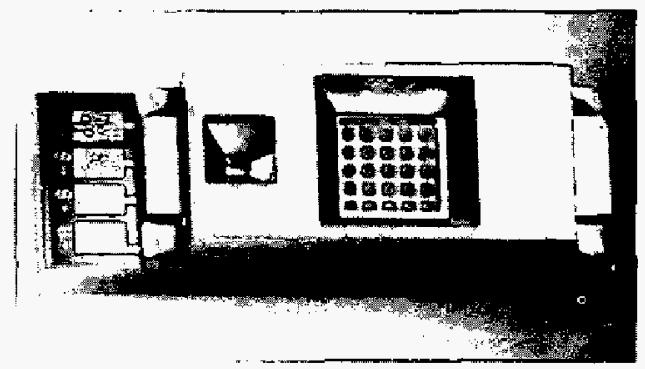

Fig. 4. Photo of the manufactured sensor Outer dimension ate $2.92 \times 0.95 \times 0.70 \mathrm{~mm}^{3}$.

The measurement setup consists of two Bronkhorst mass-flow controllers that accurately mix nitrogen and carbon dioxide with an output flow of $50 \mathrm{ml} / \mathrm{min}$ and a minimum $\mathrm{PcO}_{2}$ step of 0.5 $\mathrm{kPa}$. The $\mathrm{PcO}_{2}$ can be varied between 2 and $20 \mathrm{kPa}$, covering the medically interesting range. A temperature controller is used to maintain the temperature of the sensor constant at $37^{\circ} \mathrm{C}$.

\section{RESULTS AND DISCUSSION}

Several experiments were perforned with the hydrogel-based carbon dioxide sensor. One of the experiments is a full $\mathrm{PCO}_{2}$ sweep through the medically interesting range from 2 to $20 \mathrm{kPa} \mathrm{CO}$ with steps of $3 \mathrm{kPa} \mathrm{CO}$. The result is shown in figure 5 . Note that the initial offset pressure at 2 $\mathrm{kPa} \mathrm{CO}$ was set to zero for all experiments.

\section{TRANSDCCERS'05}

The 13th Intemational Conference on Solid-State Sensors, Actuators and Microsystems, Seoul, Korea, June 5-9, 2005 


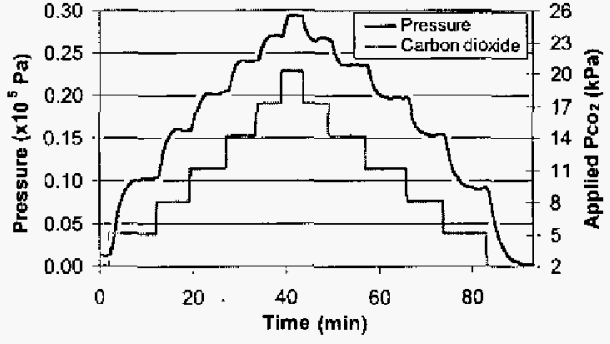

Fig. 5. Response of the sensor to a cycle from 2 to $20 \mathrm{kPa} \mathrm{CO}_{2}$ with steps of $3 \mathrm{kPa} \mathrm{CO}_{2}$ at $37^{\circ} \mathrm{C}$

As can be seen, with every increase in $\mathrm{PCO}_{2}$ the hydrogel generated an additional pressure up to $0.29 \times 10^{5} \mathrm{~Pa}$ at $20 \mathrm{kPa} \mathrm{CO}$. Stepwise decrease in $\mathrm{PCO}_{2}$ resulted in stepwise pressure decrease with very little hysteresis, which demonstrates that the process is fully reversible. For the steps up the $90 \%$ response time was determined between 2 and 4 minutes, which meets the medical requirements of far below 10 minutes.

With the results of figure 5 a plot has been constructed representing the relation between $\mathrm{PcO}_{2}$ and pressure, as shown in figure 6. By adding a fit curve the calibration curve of the sensor can be found, which is described by a second-order polynomial fit.

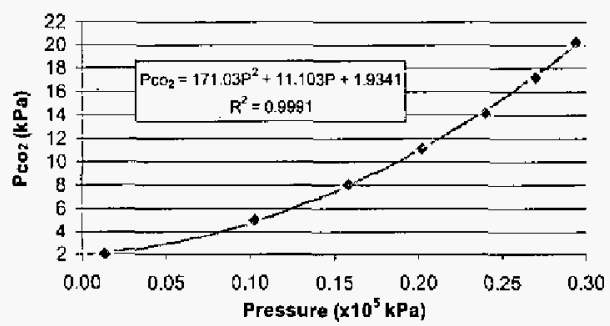

Fig. 6. Relation of the partial $\mathrm{CO}_{2}$ pressure versus the pressure and a fitted calibration curve of the sensor.

The temperature sensitivity of the sensor has also been investigated. The temperature was varied with steps of $3{ }^{\circ} \mathrm{C}$ and the equilibrium pressures were measured and plotted. The result is shown in figure 7 . The sensor appears to be linearly sensitive with the temperature. To determine the cause of the temperature sensitivity, a dummy sensor was prepared without a hydrogel. This dummy sensor showed the same behavior as presented in figure 7. Furthermore the dummy sensor also had an offset pressure, which is probably caused by stress due to the construction manner. From this the conclusion is drawn that the temperature sensitivity is caused by the constructing manner and not the $\mathrm{pH}-$ sensitive hydrogel. In future the construction method should be improved to eliminate the temperature sensitivity

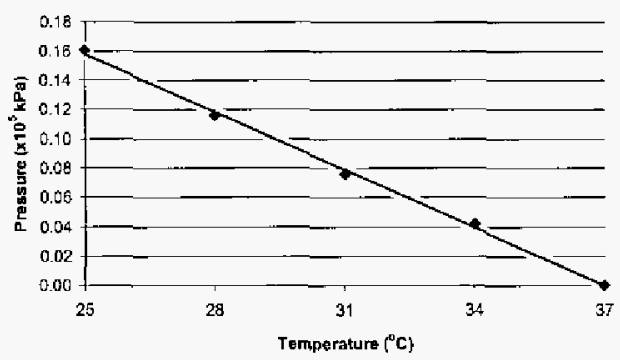

Fig. 7. The equilibrium pressures versus various temperatures.

With the used measurement setup the smallest $\mathrm{PCO}_{2}$ step possible is $0.5 \mathrm{kPa}$. It was investigated whether the sensor is capable of detecting such small steps. The result is shown in figure 8 .

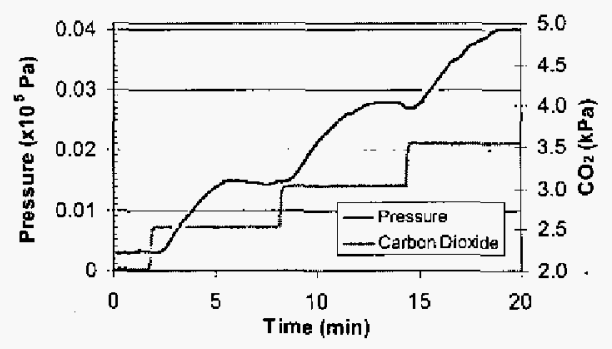

Fig. 8. Response of the sensor to carbon dioxide steps as small as $0.5 \mathrm{kPa} \mathrm{CO}_{2}$ at room temperature.

As can be seen, the pressure increased obviously with every $\mathrm{PCO}_{2}$ increase. Unfortunately, there are small fluctuations in the signal which is caused by imperfections of temperature controlling in combination with the temperature sensitivity of the sensor. Nevertheless, it is expected that the sensor is capable of detecting $\mathrm{PcO}_{2}$ steps as small as 0.1 $\mathrm{kPa}$, as required for the medical application.

The sensor should be able to resist stomach content. Hydrochloric acid can be present inside the stomach with a concentration up to $1 \mathrm{M}$. When the hydrochloric acid is able to enter the sensor through cracks or in vapor phase through the gas permeable membrane, it would titrate the $\mathrm{pH}$-sensitive hydrogel, resulting in a rapid increase in pressure generation. To investigate if hydrochloric acid has a negative influence on the sensor working, the sensor was immersed in a $1 \mathrm{M} \mathrm{HCl}$ solution while leading $2 \mathrm{kPa} \mathrm{CO} 2$ through it. The result of the experiment is shown in figure 9.

\section{TRANSDUCERS'05}

The 13th International Conference on Solid-State Sensors, Actuators and Microsystems, Seoul, Korea, June 5-9, 2005 


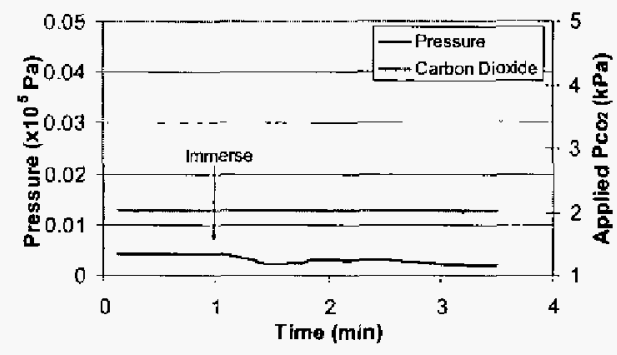

Fig. 9. Response of the scnsor to $1 \mathrm{M} \mathrm{HCl}$ at constant $2 \mathrm{kPa} \mathrm{CO}$ by immersing at $=1 \mathrm{~min}$.

At $\mathrm{t}=1$ minute the sensor was immersed. As can be seen, the sensor signal remained reasonably constant. There was only a small pressure drop which is probably caused by a small change in temperature.

\section{CONCLUSIONS}

It has been demonstrated that the presented measurement method works successfully. The hydrogel-based carbon dioxide sensor is wellcapable of measuring $\mathrm{PCO}_{2}$ within the medically interesting range of 3 to $20 \mathrm{kPa} \mathrm{CO}$. The maximum pressure generation occurred at $20 \mathrm{kPa} \mathrm{CO}_{2}$ and was $0.29 \times 10^{5} \mathrm{~Pa}$. The sensor is able to detect $\mathrm{PcO}_{2}$ changes as small as $0.5 \mathrm{kPa} \mathrm{CO}_{2}$, limited by the used measurement setup. It is assumed that the sensor can detect steps as small as $0.1 \mathrm{kPa} \mathrm{CO}_{2}$ as required for the medical application. The sensor is temperature sensitive due to the used construction mamer. However, the temperature is reasonably constant in the stomach and the temperature sensitivity can softwarematically be compensated. The sensor is able to resist up to $1 \mathrm{M}$ hydrochloric acid; nor the vapor or liquid is able to enter the sensor. From all results the conclusion is drawn that the sensor has high potential for future application.

\section{FUTURE PLANS}

In the near future clinical trials are planned. In close cooperation with Sentron Europe BV a prototype catheter with hydrogel-based carbon dioxide sensor will be constructed. At the local hospital, Twente Medisch Spectrum, facilities are available to clinically verify the working of the sensor inside the stomach. If successful, the sensor will be further developed to a medical product. However, some optimization is required such as improving the construction manner in order to decrease the temperature sensitivity. Furthermore, the thickness of the hydrogel can be decreased in order to decrease the response time. The accompanied decrease in pressure generation, and thus decrease in resolution, can be compensated by using a more sensitive pressure sensor.

\section{ACKNOWLEDGEMENTS}

Johan Bomer is gratefully acknowledged for technical support. Furthermore Ton Verloop and Jan Eijkel are thanked for useful discussions. The Dutch Technology foundation, STW, is acknowledged for financial support (project number TTF.5439). Sentron Europe BV is thanked for providing the pressure sensors and know-how.

\section{REFERENCES}

[1] A.B. Groeneveld, J.J. Kolkman, "Spanchnic Tonometry: A Review of Physiology, Methodolgy and Clinical Applications ${ }^{4 ;} J$. Crit. Care, 9, 198-210 (1994).

[2] J.J. Kolkman, "Gastric $\mathrm{Pco}_{2}$ Tonometry: Methodology and Clinical Potential for Detection of Ischemia", Thesis, Thesis Free University Amsterdam, Amsterdam, 1997.

[3] T. Tanaka, Experimental Methods in Polymer Science; $1^{\text {st }}$ ed., Academic Press, San Diego, 2000.

[4] J.H. Holtz, J.S. Holtz, C.H. Munro, S.A. Asher, "Intelligent Polymerized Crystalline Colloidal Arrays: Novel Chemical Sensor Materials", Anat. Chem, 70, 780-791 (1998).

[5] T. Tanaka, I. Nishio, S.T. Sun, S. UenoBBPPNishio, "Collapse of Gels in an Electric Field", Science, 218, 467-469 (1982).

[6] A. Suzuki, T. Tanaka, "Phase transition in polymer gels induced by visible light", Nature, 346, 345-347 (1990).

[7] H. van der Linden, S. Herber, W. Olthuis, P. Bergveld, "Stimulus-sensitive hydrogels and their applications in chemical (micro)analysis", The Analyst, 128, 325-331 (2003)

[8] J.W. Severinghaus, A.F. Bradley, "Electrodes for blood $\mathrm{PO}_{2}$ and $\mathrm{PCO}_{2}$ determination", $J$. App. Physiol., 13, 515-520 (1958).

[9] S. Herber, W. Olthuis, P. Bergveld, A. van den Berg, "A swelling hydrogel-based $\mathrm{pCO}_{2}$ sensor", Sens. Actuators B, 91, 378-382 (2003).

[10] S. Herber, W. Olthuis, P. Bergveld, A, van den Berg, "Exploitation of a $\mathrm{pH}$-sensitive hydrogel disk for $\mathrm{CO}_{2}$ detection", Sens. Actuators $B$, 103, 284-289 (2004)

[11]S. Herber, J. Eijkel, W. Olthuis, P. Bergveld, A. van den Berg, "Study of Pressure Generation of Hydrogels under Isochoric Conditions", J. Chem. Phys., 121, 2746-2751 (2004). 